\title{
Case Report: Reconstruction of a Large Maxillary Defect With an Engineered, Vascularized, Prefabricated Bone Graft
}

OPEN ACCESS

Edited by:

Giuseppe Mercante,

Humanitas University, Italy

Reviewed by:

Alberto Grammatica,

University of Brescia, Italy

Shilpi Sharma,

Narayana Superspeciality Hospital,

Gurugram, India

*Correspondence:

Ivan Martin

ivan.martin@usb.ch

tThese authors have contributed equally to this work and share first authorship

Specialty section:

This article was submitted to Head and Neck Cancer, a section of the journal

Frontiers in Oncology

Received: 13 September 2021 Accepted: 15 November 2021 Published: 06 December 2021

Citation:

Ismail $T$, Haumer A, Lunger $A$ Osinga $R$, Kaempfen A, Saxer $F$, Wixmerten A, Miot $S$, Thieringer $F$, Beinemann J, Kunz C, Jaquiéry $C$, Weikert T, Kaul F, Scherberich A, Schaefer DJ and Martin I (2021) Case Report: Reconstruction of a

Large Maxillary Defect With

an Engineered, Vascularized, Prefabricated Bone Graft.

Front. Oncol. 11:775136.

doi: 10.3389/fonc.2021.775136
Tarek Ismail ${ }^{1,2 \dagger}$, Alexander Haumer ${ }^{1,2+}$, Alexander Lunger ${ }^{1}$, Rik Osinga $^{1,3}$, Alexandre Kaempfen ${ }^{1,2}$, Franziska Saxer ${ }^{4}$, Anke Wixmerten ${ }^{2}$, Sylvie Miot ${ }^{2}$, Florian Thieringer ${ }^{5}$, Joerg Beinemann ${ }^{5}$, Christoph Kunz ${ }^{5}$, Claude Jaquiéry ${ }^{5}$, Thomas Weikert $^{6}$, Felix Kaul ${ }^{6}$, Arnaud Scherberich ${ }^{1,2}$, Dirk J. Schaefer ${ }^{1,3}$ and Ivan Martin ${ }^{2 *}$

${ }^{1}$ Department of Plastic, Reconstructive, Aesthetic and Hand Surgery, University Hospital Basel, Basel, Switzerland, 2 Department of Biomedicine, University Hospital Basel, University of Basel, Basel, Switzerland, ${ }^{3}$ Center for Musculoskeletal Infections, University Hospital Basel, Basel, Switzerland, ${ }^{4}$ Department of Orthopedic Surgery, University Hospital Basel, Basel, Switzerland, ${ }^{5}$ Clinic for Craniomaxillofacial and Oral Surgery, University Hospital Basel, Basel, Switzerland, ${ }^{6}$ Department of Radiology, University Hospital Basel, Basel, Switzerland

The reconstruction of complex midface defects is a challenging clinical scenario considering the high anatomical, functional, and aesthetic requirements. In this study, we proposed a surgical treatment to achieve improved oral rehabilitation and anatomical and functional reconstruction of a complex defect of the maxilla with a vascularized, engineered composite graft. The patient was a 39-year-old female, postoperative after left hemimaxillectomy for ameloblastic carcinoma in 2010 and tumor-free at the 5-year oncological follow-up. The left hemimaxillary defect was restored in a two-step approach. First, a composite graft was ectopically engineered using autologous stromal vascular fraction (SVF) cells seeded on an allogenic devitalized bone matrix. The resulting construct was further loaded with bone morphogenic protein-2 (BMP-2), wrapped within the latissimus dorsi muscle, and pedicled with an arteriovenous (AV) bundle. Subsequently, the prefabricated graft was orthotopically transferred into the defect site and revascularized through microvascular surgical techniques. The prefabricated graft contained vascularized bone tissue embedded within muscular tissue. Despite unexpected resorption, its orthotopic transfer enabled restoration of the orbital floor, separation of the oral and nasal cavities, and midface symmetry and allowed the patient to return to normal diet as well as to restore normal speech and swallowing function. These results remained stable for the entire follow-up period of 2 years. This clinical case demonstrates the safety and the feasibility of composite graft engineering for the treatment of complex maxillary defects. As compared to the current gold standard of 
autologous tissue transfer, this patient's benefits included decreased donor site morbidity and improved oral rehabilitation. Bone resorption of the construct at the ectopic prefabrication site still needs to be further addressed to preserve the designed graft size and shape.

Keywords: complex 3D bone defect, vascularized composite graft, bone-soft tissue interface, regenerative surgery, graft prefabrication

\section{INTRODUCTION}

Reconstruction of large bone defects in the maxillofacial region, typically relying on autologous vascularized bone grafts or synthetic biocompatible materials, remains a clinical challenge. Apart from reconstructing the hard and soft tissues, the masticatory rehabilitation of the patient by conventional (removable) or implant-supported prostheses needs to be addressed. Autologous bone grafting is associated with limited availability, significant donor site morbidity, and restrained or even impossible oral rehabilitation. Synthetic materials represent only a temporary solution, not only because of frequency of infections but also due to limited integration and thus inadequate separation of oral and nasal cavities (1-3).

The potential for de novo bone formation of cells from the stromal vascular fraction (SVF) of adipose tissue, even if not cultured or primed in vitro, has been shown in preclinical and clinical models, if implanted orthotopically (4) or exposed to low doses of bone morphogenetic protein (BMP)-2 $(5,6)$. Moreover, efficient vascularization of critically sized, SVF-based bone grafts was achieved in a rodent model by the insertion of arteriovenous (AV) bundles $(7,8)$ by analogy with typical microsurgical techniques $(9,10)$.

Here we describe the pioneering clinical implementation of an ectopically prefabricated (i.e., including a vascular pedicle for transfer) and prelaminated (i.e., multilayer composite including a soft tissue interface) (11) flap as osteogenic and vasculogenic graft for hemimaxillary reconstruction in a patient with Cordeiro type IIIa maxillectomy. The implant was first constructed in a latissimus dorsi muscle flap by combining a custom-shaped scaffold with autologous SVF cells, BMP-2, and an AV bundle. The resulting prevascularized composite graft was then transferred to the maxilla defect with the ultimate goal to durably restore midface symmetry, separate naso- and oropharyngeal spaces, allow physiological swallowing, and establish airway function.

\section{MATERIALS AND METHODS}

In this study, we report the case of a 39-year-old female patient diagnosed with an ameloblastic carcinoma of the left maxilla in September 2010. She underwent subsequent hemimaxillectomy in October 2010, which resulted in a Cordeiro type IIIa (total maxillectomy defect sparing the orbital contents) $(12,13)$, Okay Type II (14), Brown Type 3 (15) maxillectomy defect. The defect was replaced with a palatal obturator prosthesis (Figures 1A-C), which gave an unsatisfactory functional result. The orbital floor has been reconstructed with a titanium mesh (Synthes) during primary ameloblastoma resection. The patient suffered from recurrent infections and had problems drinking water due to insufficient separation of the oral and nasal cavity. After a 5-year cancer-free follow-up, the patient needed adequate and longterm surgical reconstruction of the left maxilla. The complexity of the patient's three-dimensional composite defect, in combination with her young age and her desire to bypass morbidity and limited effectiveness of a free vascularized autologous bone graft (e.g., fibula, scapula or the iliac crest), prompted for the implementation of an innovative strategy. The patient had no other relevant comorbidities. This case study conforms with the Declaration of Helsinki. It was approved by the national competent authority, Swissmedic, under exceptional permission (SBH 16-0172) and by the competent ethical committee (EKNZ), with the patient's written informed consent.

The patient's treatment consisted of a two-step reconstructive plan, namely, an ectopic implant prefabrication (Figure 1D), followed by its orthotopic transfer (Figure 1E). A threedimensional scaffold was manufactured, based on the patient's computed tomography (CT) data of the defect site and contralateral side by computer-aided design and computeraided manufacturing (CAD-CAM) techniques. As scaffolding material, allogenic and decellularized cancellous bone (Tutoplast $^{\circledR}$, Tutogen Medical, Neunkirchen, Germany) with a porosity of approximately $60 \%$ was used. The scaffold, with a total volume of $27.6 \mathrm{~cm}^{3}$, was designed as a set of four pieces, which could be intraoperatively assembled and fixed with preheated absorbable pins, leaving space for a central tunnel to allow for the intraoperative insertion of an AV bundle (Figure 1E, Figure 2B).

All surgeries were performed under general anesthesia. To collect stromal vascular fraction (SVF) cells, a hand-assisted abdominal liposuction was performed and a final volume of $320 \mathrm{ml}$ of sedimented fat was harvested. SVF cells were intraoperatively isolated with an automated device (Celution 800/CRS System, Cytori Therapeutics Inc., UK) and counted with a NucleoCounter NC-200 (ChemoMetec, Denmark), leading to a total number of $106 \times 10^{6}$ cells with a viability of $76.8+/-3.2 \%$. After assembling the four Tutoplast pieces with pins, the scaffold was cellularized by gentle loading of $105 \times 10^{6}$ SVF cells, resuspended in $8.5 \mathrm{ml}$ of fibrin gel (Tisseel ${ }^{\circledR}$, Baxter, $\mathrm{AT}$ ) in the presence of $60 \mu \mathrm{g} / \mathrm{ml}$ recombinant human bone morphogenetic protein-2 (BMP-2, Infuse ${ }^{\circledR}$, Medtronic, CH) (Figure 2A). Following SVF cell seeding, a distally ligated arteriovenous (AV) bundle (serratus branch of the 


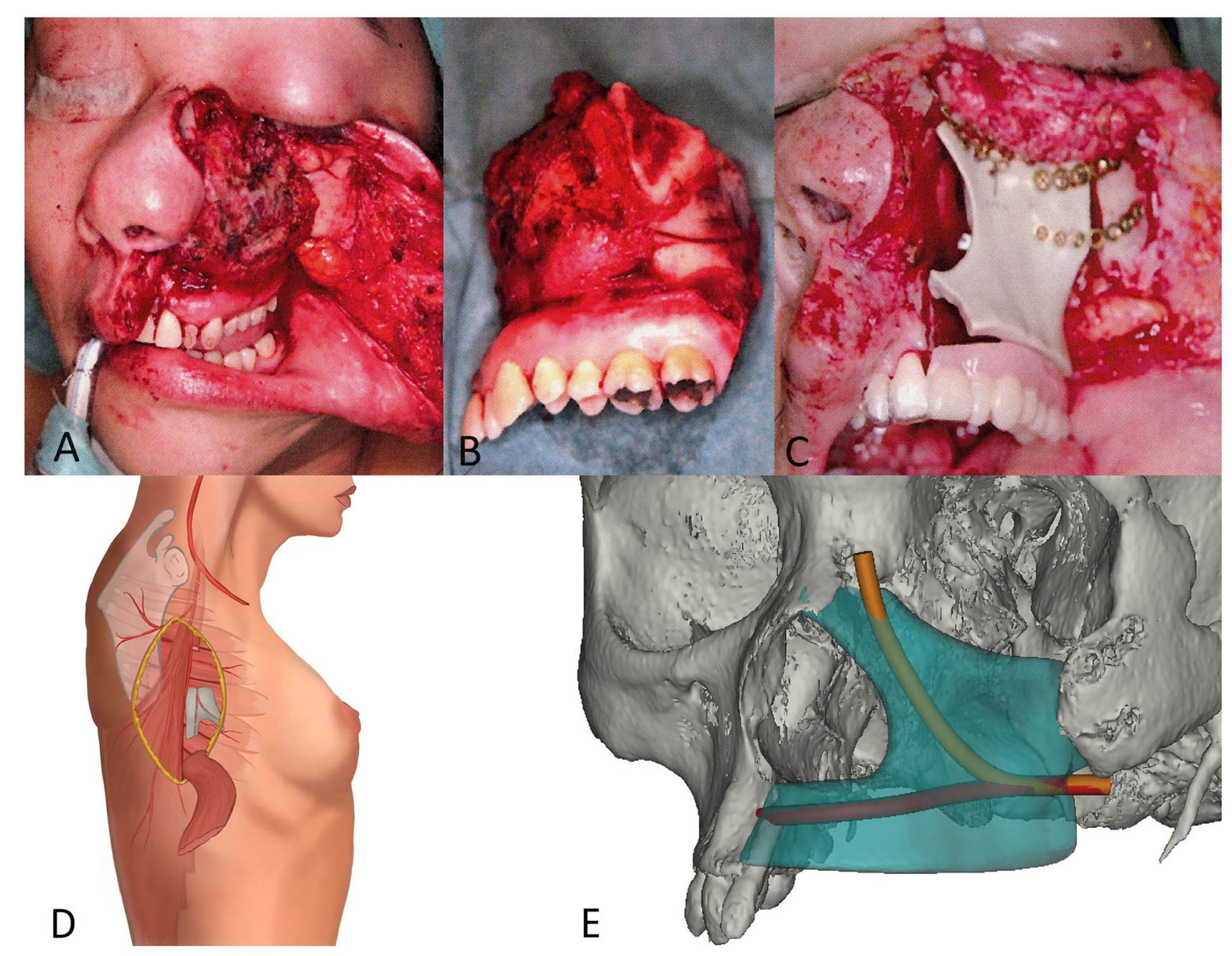

FIGURE 1 | Midface defect, immediate temporary reconstruction, and two-step patient specific planning. (A) Intraoperative aspect of exposed ameloblastic carcinoma of the left hemimaxilla prior to resection. (B) Left hemimaxillectomy specimen with preservation of midface soft tissue and missing inner nasal lining (mucosa). (C) Intraoperative situation after PEEK implantation with obturator prosthesis. (D) Ectopic prevascularization by surgical insertion of an arteriovenous (AV) bundle (serratus branch of the thoracodorsal vessel) and wrapping of the construct into a split latissimus muscle. (E) CAD-CAM reconstruction on the patients CT scan showing the customized Tutoplast ${ }^{\circledR}$ scaffold with the tunnel planned for the serratus AV bundle and its branching after implantation.

thoracodorsal vessels) was surgically inserted in the central scaffold tunnel for axial prevascularization (Figure 2B). The whole construct was wrapped in a split latissimus dorsi muscle flap and placed under the patient's left breast (Figures 2C, D). Total surgery time was $6 \mathrm{~h}$ and $30 \mathrm{~min}$, while flap raise including vessel dissection and ectopic construct preparation (fixation of the scaffold parts, SVF isolation, seeding of the scaffold) was performed simultaneously.

Vascularization of the construct was analyzed by perfusion magnetic resonance imaging (MRI) after 1 and 6 weeks. Bone metabolism inside the construct was assessed with single-photon emission computed tomography (SPECT) after 6 weeks.

After 8 months and proven neovascularization with manifest bone metabolism as seen on the SPECT scan, the composite graft was harvested and positioned into the recipient site, while a punch biopsy $(4 \times 5 \mathrm{~mm})$ was taken from the central volume for histological analysis. The thoracodorsal vessels were dissected free, cut, and anastomosed in an end-to-end fashion to the left facial artery and vein. Graft vascularization after transfer was intraoperatively assessed by fluorescent indo cyanine green (ICG) visualization (VisionSense, Philadelphia). The donor site was then settled by primary closure. Postoperative monitoring at the intensive care unit (ICU) was uneventful. Total surgery time was $8 \mathrm{~h} 18 \mathrm{~min}$. The patient was hospitalized for 7 days. Suction drains at the donor site were removed on the third postoperative day. Antibiotic therapy with amoxicillin/clavulanic acid for a total of 5 days was administered perioperatively.

Regular follow-up appointments were scheduled at 2 and 6 weeks as well as 3, 6, and 12 months post-reconstruction. Final imaging by CT scan and logopedic evaluation to assess speech and swallowing function were conducted 24 months after orthotopic transfer. 


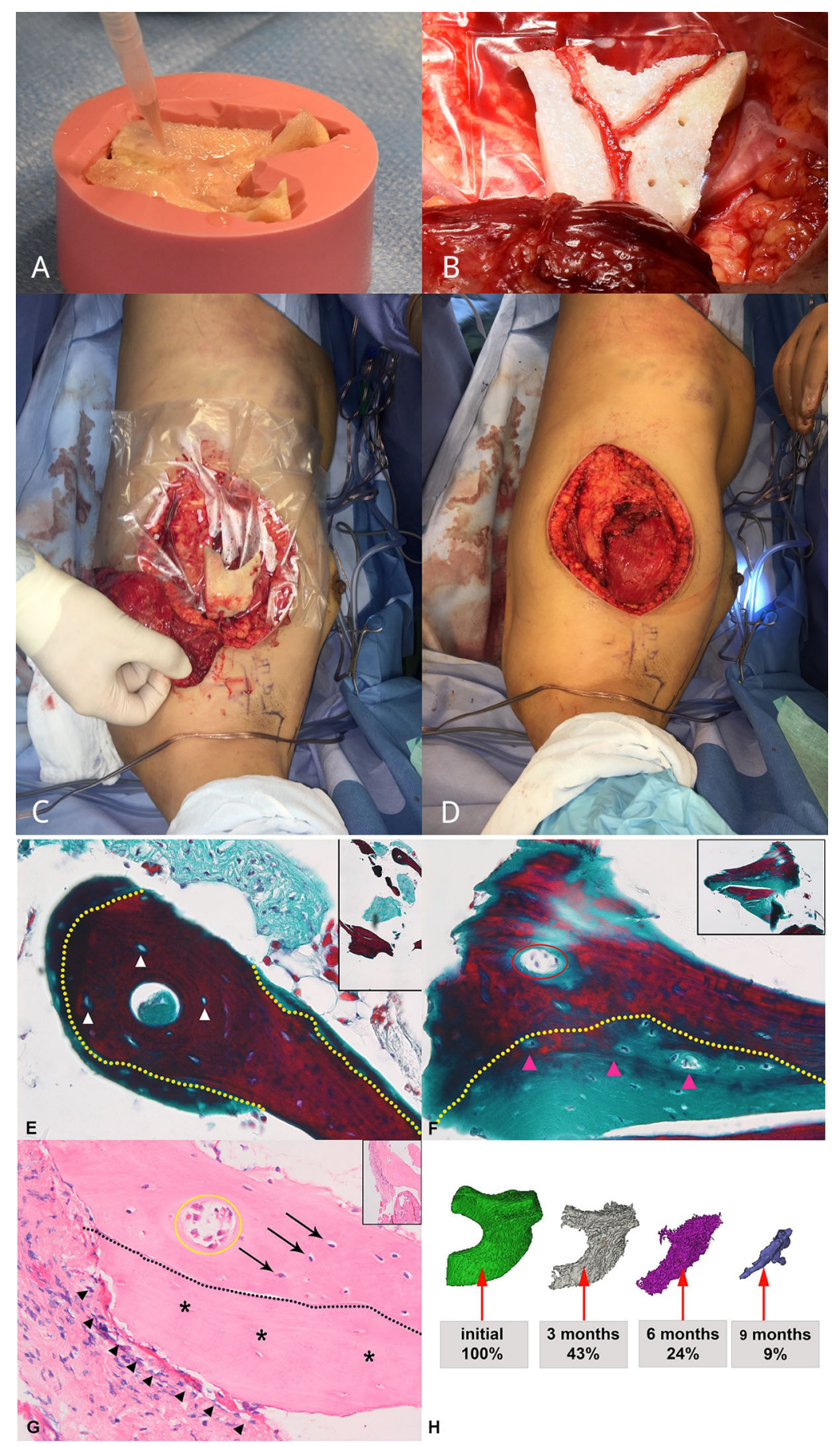

FIGURE 2 | Graft prefabrication and histological analysis (bone biopsy at moment of transfer at week 32) and volume assessment over time. (A) 3D scaffold of devitalized bone manufactured to match the patient's defect size and shape and seeded with SVF cells and BMP-2. (B) Ectopic implantation with serratus AV bundle. (C, D) Construct with vascular pedicle before and after being wrapped in split latissimus muscle. (E, F) High magnification of representative figures of bone biopsy after staining with Masson Trichrome. The Tutoplast ${ }^{\circledR}$ scaffold is characterized by purple staining, representing mature bone and cellular lacunae (white arrowheads), showing devitalized bone tissue. Newly formed bone tissue, represented by light green color, is deposited on the Tutoplast ${ }^{\circledR}$ scaffold and contains nuclei (pink arrowheads). The yellow dashed line delineates the original scaffold material and apposition of newly formed bone. A vessel (red circle) demonstrates that the scaffold is vascularized. (G) Overview figure shows appositional bone growth on the Tutoplast ${ }^{\circledR}$ scaffold (asterisk). Osteocytes (arrows) are visible in the newly formed bone. The proportion of scaffold vs. new bone formation is close to 50:50. A blood vessel is present within the newly formed bone (yellow circle). Osteoclasts (full arrowheads) fringe the Tutoplast ${ }^{\circledR}$ scaffold (asterisks), which shows clear signs of degradation at site of interaction. There is no major osteoclast infiltration at the level of the newly formed apposed bone and no sign of degradation visible. (H) CT-reconstruction and volume calculation show volume decrease over time. 


\section{RESULTS}

\section{Ectopic Implant Prefabrication}

The patient had an uneventful course after ectopic graft placement in December 2016, without intraoperative complications such as major bleeding or pedicle damage. The patient did not report any implant-related discomfort, and neither hematoma nor infection was noted.
Vascularization of the construct was assessed during ectopic graft development after 1 week (Figure 3A) and 6 weeks (Figure 3B) by dynamic contrast-enhanced MR perfusion imaging. The AV bundle proved to be patent, providing intrinsic perfusion to the construct. The latissimus dorsi muscle wrap was viable, with a perfusion pattern comparable or superior to the subscapularis muscle, chosen as a reference for physiological perfusion in this anatomic region. At both time
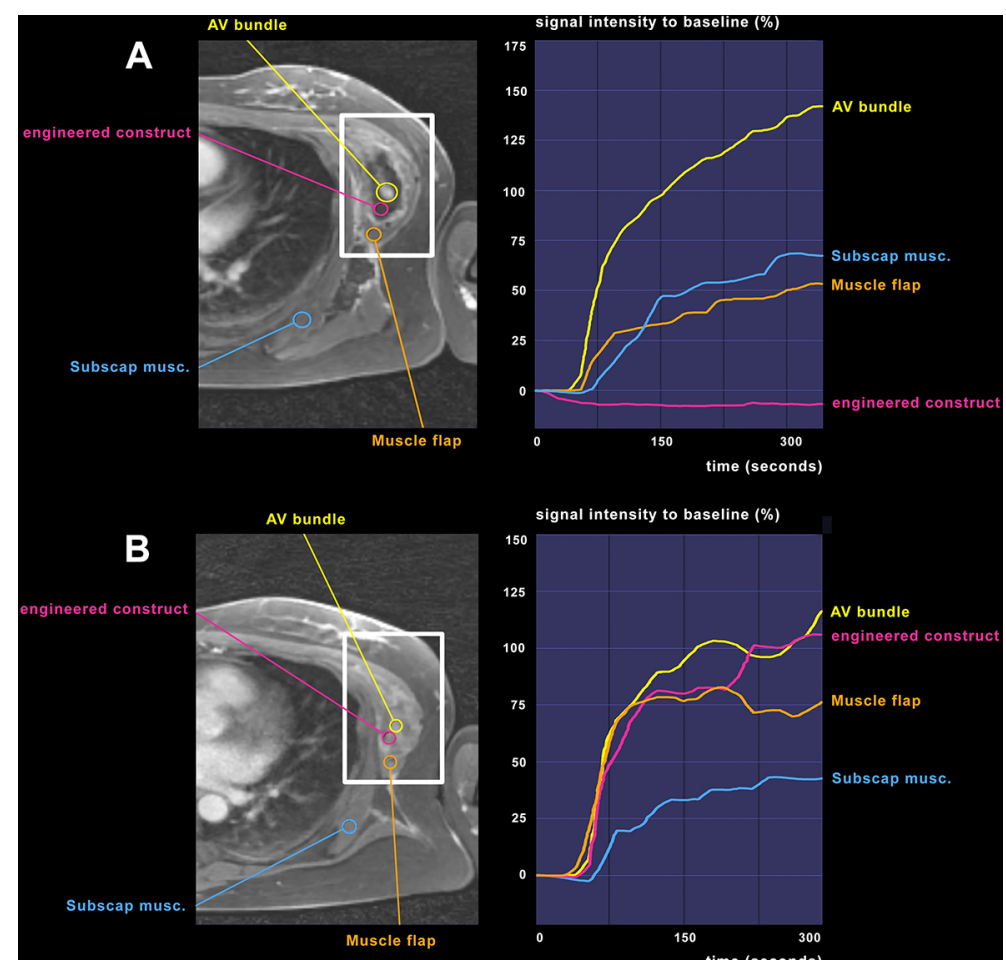

signal intensity to baseline $(\%)$

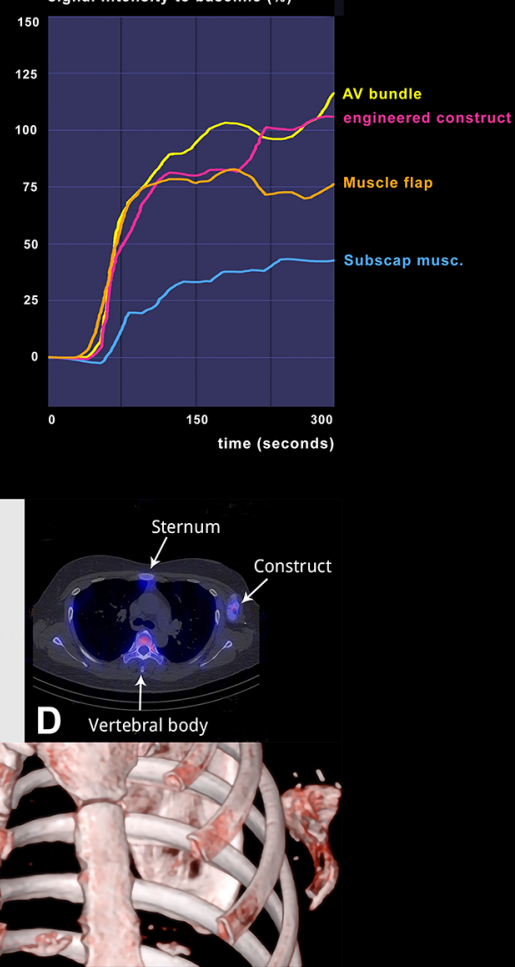

FIGURE 3 | Longitudinal assessment of vascularization and bone formation. Transversal images of a golden-angle radial sparse parallel (GRASP) MRI showing the perfusion of the engineered construct adjacent to the left ribcage over time as well as corresponding signal-time curves at 1 week (A) and at 6 weeks (B). The Maxilla construct is highlighted by white rectangles. Signal intensity in relation to $t=0$ (injection of contrast agent) on the $Y$-axis, time in seconds on the $X$-axis. Violet region of interest $(\mathrm{ROI})$ located in the construct, blue $\mathrm{ROI}$ located in the left $\mathrm{M}$. subscapularis in situ and yellow $\mathrm{ROI}$ encompassing the $\mathrm{AV}$ bundle. Orange $\mathrm{ROI}$ indicates M. latissimus dorsi flap covering the construct. M. subscapularis was used as a reference for physiological vascularization in this area. Planar images (C) and SPECT and SPECT/CT images (D) after 6 weeks of the bone scintigraphy, showing strong DPD uptake in the construct, indicating bone turnover and vitality of the construct. In a region-of-interest (ROI) analysis of the maxilla construct at its largest diameter in comparison to the sternum (as a bone with similar structure/size). (E) VRT (volume rendering technique) from the SPECT illustrates the location of the prefabricated construct. 
points, the subscapularis muscle, the latissimus muscle, and the AV bundle showed a normal, steadily increasing perfusion over time. When focusing on the engineered construct, no signal increase after injection of the contrast agent was observed after 1 week, whereas a steep increase in signal intensity was visible after 6 weeks, demonstrating functional internal vascularization.

Bone metabolism of the construct was assessed after 6 weeks by SPECT/CT (Figures 3C-E). Active 3,3-diphosphono-1,2propanodicarboxylic acid (DPD) uptake in the construct revealed traits of viable bone tissue (maximum counts: 112), in the range between the sternal bone (maximum counts: 95) and the highly compact thoracic vertebral body (maximum counts: 154), used as reference structures of similar size and position (Figures 3C, D). Bone biopsy at the phase of construct transfer, 32 weeks after ectopic implantation, morphologically confirmed de novo bone formation (Figures $\mathbf{2 E - G}$ ). Masson Trichrome staining showed a clear distinction between the Tutoplast ${ }^{\circledR}$ scaffold material, consisting of devitalized bone, and newly deposited bone matrix. The latter was less dense and less mature, as shown by the green color and reduced lamellar structure. The deposited bone contained stained cell nuclei as sign of living tissue, clearly distinguishable from the devitalized bone of the scaffold, where cells were visible as empty lacunae. Vascularization of the construct was histologically verified by piercing vessels in the scaffold material (Figures 2F, G). Osteoclasts were found around the Tutoplast ${ }^{\circledR}$ scaffold, which displayed morphological signs of active resorption. Within the newly formed, apposed bone, there was no major osteoclast infiltration and no signs of degradation. Quantitative histomorphometry of the biopsy specimen indicated an average of $30.2 \%(\mathrm{SD}=10.3)$ of newly formed bone over the total bone area.

Mineralized tissue volume in the construct was assessed by CT scan at 3, 6, and 9 months postoperatively (Figure $\mathbf{2 H}$ ). Progressive resorption of the mineralized mass was observed, down to $43 \%, 24 \%$, and $9 \%$ of the initial scaffold volume.

\section{Orthotopic Transplantation}

After 32 weeks, despite advanced resorption, the ectopically engineered composite graft was transferred into the maxillary defect. It was anastomosed to the facial artery and external jugular vein as a free tissue transfer (Figures 4A, B). The graft was augmented with calvarial bone struts from the patient's left parietal region in order to compensate for the loss of bone volume from the initial design. The postoperative course was uneventful. After one night in the ICU, the patient was transferred to the general ward and dismissed after 7 days. Wound healing and flap integration were satisfactory.

After additional 24 months, oral rehabilitation was evaluated with a clinical assessment of the swallowing and speech function as well as a palatogram and myometric measurement of the lips, tongue, masseter muscle, and mentalis muscle by a speech therapist. The patient yielded a near-to-normal oropharyngeal function with normal speech. She was able to eat an unrestricted diet, presented no ectropion, enophthalmos, or diplopia corresponding to normal globe position and function. No microstomy was observed, and oral competence was restored including normal tongue movement, mouth opening, and oral and lip sensation (Table 1). Although the patient reported increased trapezius and suboccipital muscle tonicity and slight drooling on the left side, facial symmetry was achieved with aesthetic satisfaction, based on self- and clinical assessment. The CT scan 24 months after orthotropic transfer showed a resorption of the construct with a remaining bone core (Figures 4C-H).

Symmetry and subjective aesthetic outcome were assessed after reconstruction (Figure 5).

\section{DISCUSSION}

This case describes the unique combination of bone tissue engineering, ectopic prefabrication, and microvascular free-flap strategies for the reconstruction of a highly complex defect of the left hemimaxilla in a young female patient. With this concept, we were able to create a prefabricated, composite maxilla substitute providing both bone and a soft tissue interface, thus avoiding the use of a non-autologous structural support material, such as a titanium cage, and the morbidity associated with autologous vascularized bone tissue transfer.

It is known that midface defects, especially of the maxilla, can have a substantial functional impact. Conventional reconstructive options, including freely transferred or local tissue flaps, typically fail to provide a sufficient anatomical and functional coverage for defects involving the hard and soft palate, as well as the orbital floor $(19,20)$. This leads to inadequate mastication, food processing, and lack of separation between the oral and nasal cavities. Traditionally, combinations of non-vascularized bone grafts with myocutaneous free flaps as well as free osteomyocutaneous flaps have been used for the coverage of these defects, all of them with specific advantages and disadvantages. Several chimeric free flaps have been described from the fibula or scapula with skin island. Extensive defects involving the palate and orbital floor, as in the patient described, have a better functional outcome and quality of life if reconstructed with free flaps, compared to prostheses. Still, this is a valuable option in mild to moderate-sized palatal defects or in elderly, multimorbid patients $(21,22)$. Free flaps in general inherently cause a donor site defect and associated complications. In free fibula bone flaps, which are often used in maxillofacial reconstruction, perioperative donor site complications occur in about one-third of cases and long-term morbidities of $17 \%$ have been described, including leg weakness, ankle instability, hallux contracture, and decreased ankle mobility (23). Other osteocutaneous flaps, such as the iliac crest flap, can lead to a sensory deficit (up to 27\%), chronic pain (8\%-26\%) (24, $25)$, or impaired gait and reduced range of hip motion (25\%) (26). For the radial forearm osteocutaneous flap, wound breakdown with tendon exposure is known to occur in $5 \%-46 \%(27,28)$, whereas fracture of the residual radius occurs in $0 \%-18 \%(28-30)$ and chronic pain in $16.7 \%$ (31).

To avoid harvesting large amounts of autologous bone tissue, we decided to combine strategies of tissue engineering with approaches of plastic and reconstructive surgery, implementing 


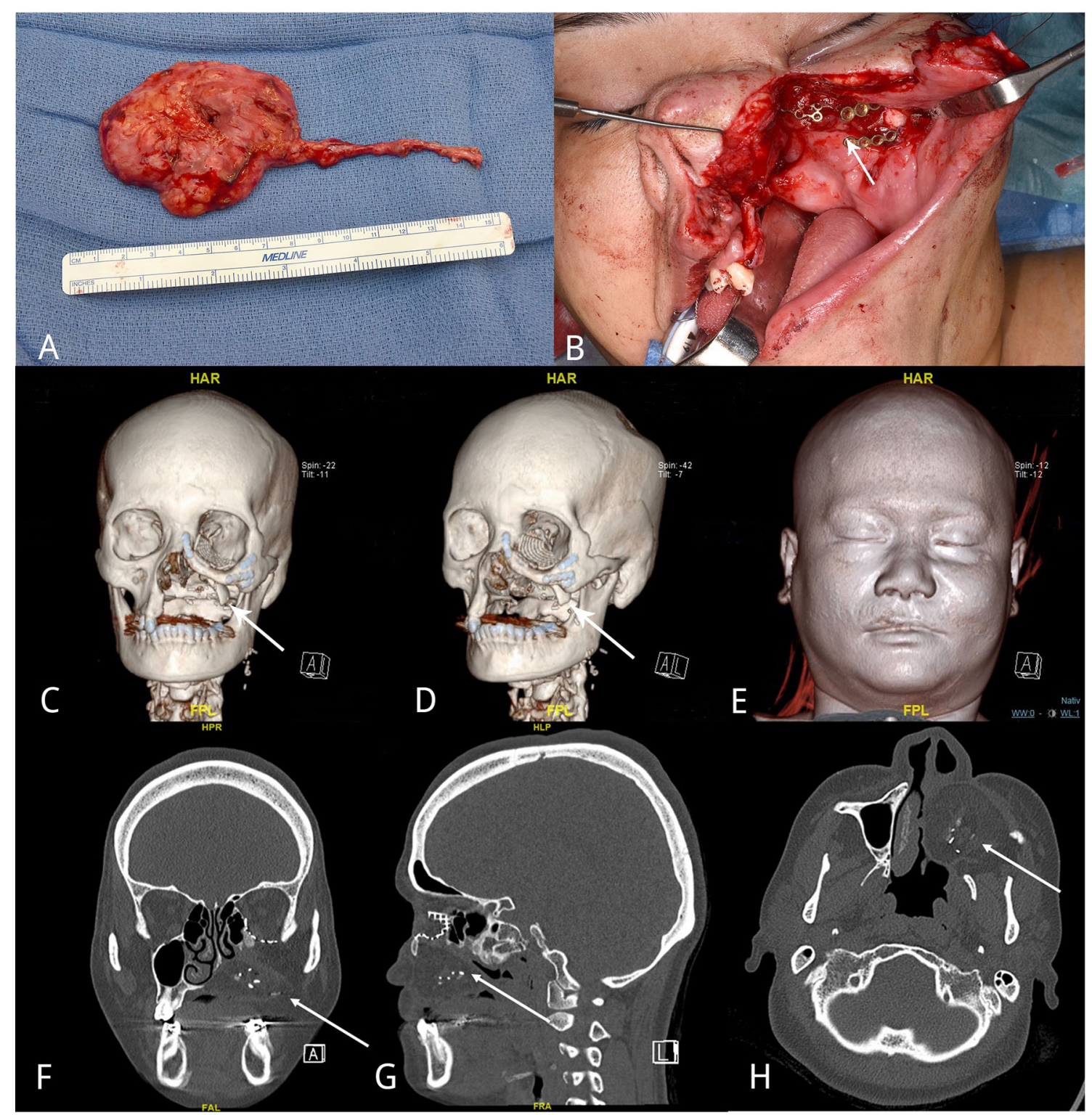

FIGURE 4 | Orthotopic graft transfer and follow-up imaging. Free tissue transfer of the engineered bone-soft tissue composite was performed 9 months after the first step of prefabrication. (A) Harvesting of the composite graft, consisting of wrapped latissimus dorsi muscle and the engineered vital bone germ and the AVbundle. (B) Transfacial incision. Exposure of the defect after removal of PEEK implant. (C, D) Tabula externa (white arrows) struts served as a substitute for missing bone parts in order to reconstruct the infraorbital rim. (E) 3D rendering with symmetric soft tissue coverage. Follow-up CT-imaging 24 months after the orthotopic transfer of the graft from $\mathbf{( F )}$ coronary, $(\mathbf{G})$ sagittal, and $\mathbf{( H )}$ transverse views. The arrows point to the reconstructed bone tissue present after 24 months.

a "regenerative surgery" paradigm (32). The bone-forming capacity of the engineered graft was based on three traditional osteogenic principles (33), namely, (i) osteoconduction, provided by the allogenic, devitalized scaffolding material, (ii) osteogenesis, through the patient's own osteoprogenitor cells derived from the intraoperatively gained adipose stromal vascular fraction (SVF), and (iii) osteoinduction, through the delivery of BMP-2. The intraoperative tissue engineering approach was substantiated by previous preclinical and clinical studies $(4,5)$ and bypassed complex, time-consuming and costly in vitro cell culture. The engineered graft, ectopically prefabricated with an associated AV bundle, achieved efficient vascularization and bone formation within a confined space. Moreover, its composite nature, including an interface with soft tissues, enabled to restore the patient's anatomical and functional deficit, to provide support to the eye globe, to obliterate the communication between the orbit and the nasopharynx, and to reconstruct the palatal surface.

Table 1 shows in detail the clinical advantages of the developed technique as compared to the expected outcome 
TABLE 1 | Comparison of the functional outcome between standard of care with autologous tissue transfer and experimental procedure.

\begin{tabular}{|c|c|c|c|c|}
\hline $\begin{array}{l}\text { Outcome } \\
\text { measures }\end{array}$ & $\begin{array}{l}\text { Experimental procedure } \\
\text { (described case) }\end{array}$ & $\begin{array}{c}\text { Standard procedure }(\%) \\
\text { [Cordeiro PG (16)] }\end{array}$ & $\begin{array}{c}\text { Standard procedure (\%) } \\
\text { [Moreno MA (17)] }\end{array}$ & $\begin{array}{c}\text { Standard procedure (\%) } \\
\text { [Sweeny AR (18)] }\end{array}$ \\
\hline \multicolumn{5}{|l|}{ Speech } \\
\hline Normal & $\checkmark$ & 50 & 47.5 & \\
\hline Nearly normal & $x$ & 34.1 & 40 & \\
\hline Intelligible & $x$ & 13.6 & 7.5 & \\
\hline Unintelligible & $x$ & 2.3 & 5 & \\
\hline \multicolumn{5}{|l|}{ Diet } \\
\hline Unrestricted & $\checkmark$ & 52 & 55 & \\
\hline Soft & $x$ & 42 & 35 & \\
\hline Liquids & $x$ & 6 & 5 & \\
\hline Feeding tube & $x$ & 2 & 5 & \\
\hline \multicolumn{5}{|l|}{$\begin{array}{l}\text { Globe Position and } \\
\text { function }\end{array}$} \\
\hline Normal & $\checkmark$ & 23.8 & & \\
\hline Dystopia & $x$ & 4.8 & & \\
\hline Diplopia & $x$ & 19 & & 8 \\
\hline Enophthalmos & $x$ & 4.8 & & \\
\hline Ectropion & $x$ & 47.6 & & 50 \\
\hline Epiphora & $x$ & & & 29 \\
\hline Exposure & $x$ & & & 25 \\
\hline \multicolumn{5}{|l|}{ keratopathy } \\
\hline Lagopthalmos & $x$ & & & 16 \\
\hline Fistula & $x$ & & & 8 \\
\hline Midface deformity & $x$ & & & 4 \\
\hline \multicolumn{5}{|l|}{ Oral competence } \\
\hline Yes & $\checkmark$ & 91.7 & & \\
\hline No & $x$ & 8.3 & & \\
\hline Drooling & $\checkmark$ & & & \\
\hline \multicolumn{5}{|l|}{ Microstomia } \\
\hline Yes & $x$ & 25 & & \\
\hline No & $\checkmark$ & 75 & & \\
\hline \multicolumn{5}{|l|}{ Aesthetic results } \\
\hline Excellent & $\checkmark$ & 58.6 & & \\
\hline Good & $x$ & 35.7 & & \\
\hline Fair & $x$ & 5.7 & & \\
\hline Poor & $x$ & 0 & & \\
\hline
\end{tabular}

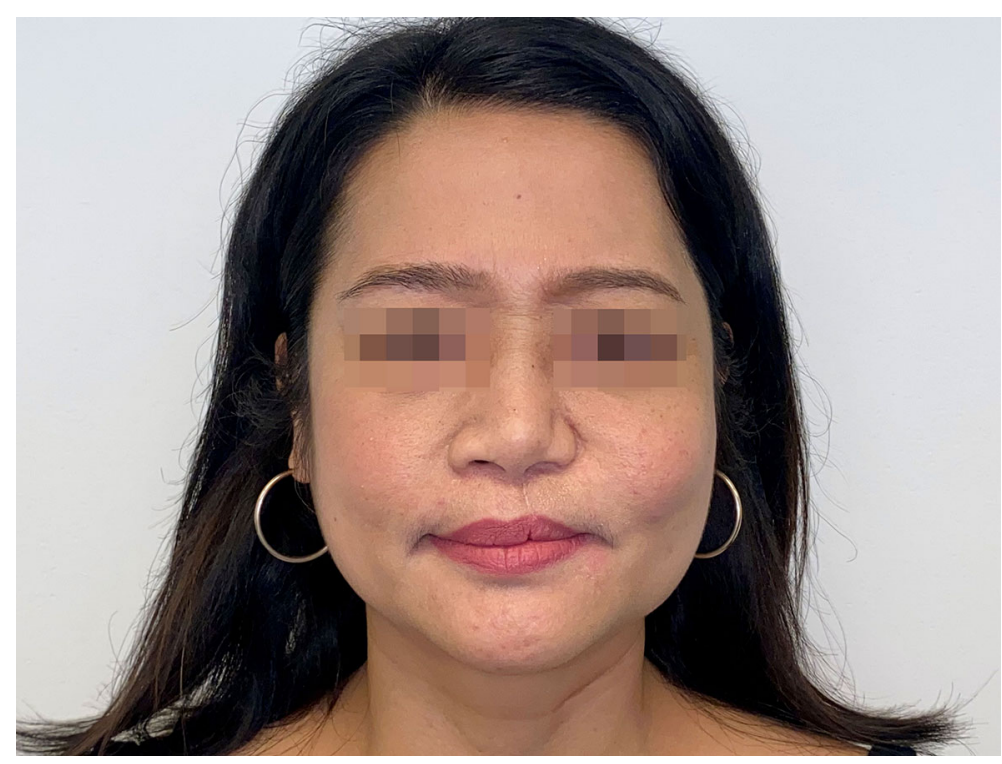

FIGURE 5 | Photographic documentation after reconstruction shows symmetrical and aesthetically pleasing result. 
following the standard of care described by Moreno (17), Cordeiro (16), or Sweeney (18). Moreover, in contrast to previously described cases of tissue engineering-based mandibular defect reconstructions, we were able to avoid foreign material (titanium cage), as used by Warnke et al. and Wiltfang et al. $(34,35)$. By doing so, we avoided not only the postoperative risk of developing an implant-associated infection which, for titanium implants, is reported to be around $7 \%$ but also the life-long risk of later hematogenous infection of the implant (36) and the so-called foreign body reactions, where the implant is encapsulated and cannot be integrated with the surrounding tissue (37).

In contrast to previous studies, we used SVF cells from adipose tissue instead of bone-marrow mesenchymal stromal cells (BM-MSC). Adipose stromal cells have recently emerged as a viable source for clinical applications, because of their abundance and easy access. When compared to BM-MSC, SVF cells do require an osteogenic priming, here offered by the BMP2 delivery, but display higher resistance to hypoxia-induced apoptosis and oxidative stress-induced senescence and have more potent proangiogenic activity (38-41).

The main limitation of the developed procedure was a significant resorption of the original scaffold material during the construct prefabrication, so that a sufficient bone stock could not be maintained for dental implants, representing the ultimate solution for mastication and phonation. One possible explanation for the observed resorption might be due to an effect of the supplied BMP-2. Besides their osteoinductive properties, BMPs are known to invoke a seemingly dosedependent allograft resorption mediated by osteoclasts. Pradhan et al. reported that BMP-2 treatment of a bone graft might cause a higher non-union rate compared with nontreatment, which was attributed to an aggressive boneresorptive phase prior to osteoinduction (42). Similarly, Vaidya et al. showed that BMP-2-treated bone grafts for spinal fusion lost their original height and structure, likely due to activated bone resorption (43). In addition, Seeherman et al. recently reported that treatment with BMP-2 in a primate bone defect model increased the size of the defect and the number of osteoclasts, so that the expected bone formation was preceded by bone resorption (44). These clinical studies are consistent with experimental evidences that BMP-2 and BMP-7 may reduce bone size by directly or indirectly activating osteoclasts (45). Other possible explanations for the bone resorption observed in the present case could be related to the missing mechanical load during the ectopic prefabrication phase (46) or to the biological influence of the muscle tissue in direct contact with the construct. In fact, it was reported that in calcium sulfate/ apatite bone substitutes with direct contact to muscle, the calcium sulfate phase was resorbed after 6 weeks and the hydroxyapatite content decreased significantly over time (47).

Further studies are therefore required to identify the underlying cause of bone resorption and to possibly counteract it. For example, combined anti-resorptive therapies, such as bisphosphonates, have been preclinically validated in a variety of bone repair models, especially to reduce BMP catabolic effects
(48-51), and may be considered in future developments. More bone mass would allow the patient to receive tooth implants after the primary procedure and without need of additional bone transfer to the defect site.

Despite this limitation, the engineered graft and its surgical implementation achieved a separation between the oral and nasal cavities in the setting of a critical maxilla defect and provided structural support to the orbital floor. This was of substantial benefit to the patient, without drawbacks of free vascularized autologous bone grafting procedures or foreign body implantation. The clinical case thus represents a proof of principle for a "regenerative surgery"-based prefabrication concept and warrants consideration for reconstruction of complex composite defects in functionally and aesthetically highly demanding areas.

\section{DATA AVAILABILITY STATEMENT}

The original contributions presented in the study are included in the article/supplementary material. Further inquiries can be directed to the corresponding author.

\section{ETHICS STATEMENT}

The studies involving human participants were reviewed and approved by Swissmedic, under exceptional permission (SBH 160172) of the ethical committee (EKNZ). The patients/ participants provided their written informed consent to participate in this study.

\section{AUTHOR CONTRIBUTIONS}

$\mathrm{TI}$ and $\mathrm{AH}$ were responsible for the conception and design, acquisition of data, analysis and interpretation of data, and writing and revision of the manuscript. TI, AH, DS, AL, AK, FT, CK, and CJ performed the surgery. AL, RO, AK, FT, and FS contributed to the analysis and interpretation of data and revision of the manuscript. JB conceived and designed the analysis, collected the data, and contributed data or analysis tools. TW and FK contributed to the conception and design, acquisition of data, and analysis and interpretation of data. AS, DS, and IM provided senior advice, counseling, writing support, and revision of the manuscript. AW and SM provided conception and design and regulatory aspects. All authors contributed to the article and approved the submitted version.

\section{FUNDING}

This work was supported by the Propatient Forschungsstiftung, University Hospital Basel. 


\section{REFERENCES}

1. Järvinen S, Suojanen J, Kormi E, Wilkman T, Kiukkonen A, Leikola J, et al. The Use of Patient Specific Polyetheretherketone Implants for Reconstruction of Maxillofacial Deformities. J Cranio-Maxillofac Surg (2019) 47:1072-6. doi: 10.1016/j.jcms.2019.03.018

2. Alonso-Rodriguez E, Cebriàn JL, Nieto MJ, Del Castillo JL, Hernàndez-Godoy J, Burgueño M. Polyetheretherketone Custom-Made Implants for Craniofacial Defects: Report of 14 Cases and Review of the Literature. J Cranio-Maxillofac Surg (2015) 43:1232-8. doi: 10.1016/j.jcms.2015.04.028

3. Jonkergouw J, van de Vijfeijken SECM, Nout E, Theys T, Van de Casteele E, Folkersma H, et al. Outcome in Patient-Specific PEEK Cranioplasty: A TwoCenter Cohort Study of 40 Implants. J Cranio-Maxillofac Surg (2016) 44:1266-72. doi: 10.1016/j.jcms.2016.07.005

4. Saxer F, Scherberich A, Todorov A, Studer P, Miot S, Schreiner S, et al. Implantation of Stromal Vascular Fraction Progenitors at Bone Fracture Sites: From a Rat Model to a First-In-Man Study. Stem Cells (2016) 34:2956-66. doi: $10.1002 /$ stem. 2478

5. Mehrkens A, Saxer F, Güven S, Hoffmann W, Müller AM, Jakob M, et al. Intraoperative Engineering of Osteogenic Grafts Combining Freshly Harvested, Human Adipose-Derived Cells and Physiological Doses of Bone Morphogenetic Protein-2. Eur Cells Mater (2012) 24:308-19. doi: 10.22203/ eCM.v024a22

6. Jeon O, Song SJ, Kang SW, Putnam AJ, Kim BS. Enhancement of Ectopic Bone Formation by Bone Morphogenetic Protein-2 Released From a HeparinConjugated Poly(L-Lactic-Co-Glycolic Acid) Scaffold. Biomaterials (2007) 28:2763-71. doi: 10.1016/j.biomaterials.2007.02.023

7. Ismail T, Osinga R, Todorov AJ, Haumer A, Tchang LA, Epple C, et al. Engineered, Axially-Vascularized Osteogenic Grafts From Human AdiposeDerived Cells to Treat Avascular Necrosis of Bone in a Rat Model. Acta Biomater (2017) 63:236-45. doi: 10.1016/j.actbio.2017.09.003

8. Epple C, Haumer A, Ismail T, Lunger A, Scherberich A, Schaefer DJ, et al. Prefabrication of a Large Pedicled Bone Graft by Engineering the Germ for De Novo Vascularization and Osteoinduction. Biomaterials (2019) 192:118-27. doi: 10.1016/j.biomaterials.2018.11.008

9. Tanaka Y, Sung K-C, Tsutsumi A, Ohba S, Ueda K, Morrison W. Tissue Engineering Skin Flaps: Which Vascular Carrier, Arteriovenous Shunt Loop or Arteriovenous Bundle, has More Potential for Angiogenesis and Tissue Generation? Plast Reconstr Surg (2003) 112:1636-44. doi: 10.1097/ 01.PRS.0000086140.49022.AB

10. Erol OO, Sira M. New Capillary Bed Formation With a Surgically Constructed Arteriovenous Fistula. Plast Reconstr Surg (1980) 66:109-15. doi: 10.1097/ 00006534-198007000-00021

11. Pribaz JJ, Fine NA. Prefabricated and Prelaminated Flaps for Head and Neck Reconstruction. Clin Plast Surg (2001) 28:261-+. doi: 10.1016/S0094-1298(20) 32362-2

12. Halvorson EG, Cordeiro PG, Disa JJ, Wallin EF, Mehrara BJ. Superficial Temporal Recipient Vessels in Microvascular Orbit and Scalp Reconstruction of Oncologic Defects. J Reconstr Microsurg (2009) 25:383-7. doi: 10.1055/s0029-1220859

13. Disa JJ, Cordeiro PG. Mandible Reconstruction With Microvascular Surgery. Semin Surg Oncol Vol (2000) 19:226-34. doi: 10.1002/1098-2388(200010/11) 19:3<226::AID-SSU4>3.0.CO;2-N

14. Okay DJ, Genden E, Buchbinder D, Urken M. Prosthodontic Guidelines for Surgical Reconstruction of the Maxilla. J Prosthet Dent (2001) 86:352-63. doi: 10.1067/mpr.2001.119524

15. Brown JS, Rogers SN, McNally DN, Boyle M. A Modified Classification for the Maxillectomy Defect. Head Neck (2000) 22:17-26. doi: 10.1002/(SICI)10970347(200001)22:1<17::AID-HED4>3.0.CO;2-2

16. Cordeiro PG, Chen CM. A 15-Year Review of Midface Reconstruction After Total and Subtotal Maxillectomy: Part I. Algorithm and Outcomes. Plast Reconstruct Surg (2012). doi: 10.1097/PRS.0b013e318221dca4

17. Moreno MA, Skoracki RJ, Hanna EY, Hanasono MM. Microvascular Free Flap Reconstruction Versus Palatal Obturation for Maxillectomy Defects. Head Neck (2010). doi: 10.1002/hed.21264

18. Sweeney AR, Walker B, Bhrany AD, Chang SH, Jian-Amadi A. Ophthalmic Changes Following Maxillectomy With or Without Postoperative Radiation Therapy. J Craniofac Surg (2019). doi: 10.1097/SCS.0000000000005437
19. Andrades P, Militsakh O, Hanasono MM, Rieger J, Rosenthal EL. Current Strategies in Reconstruction of Maxillectomy Defects. Arch Otolaryngol Head Neck Surg (2011) 137:806-12. doi: 10.1001/archoto.2011.132

20. Spiro RH, Strong EW, Shah JP. Maxillectomy and its Classification. Head Neck (1997) 19:309-14. doi: 10.1002/(SICI)1097-0347(199707)19:4<309:: AID-HED9>3.0.CO;2-4

21. Genden EM, Rinaldo A, Suárez C, Wei WI, Bradley PJ, Ferlito A. Complications of Free Flap Transfers for Head and Neck Reconstruction Following Cancer Resection. Oral Oncol Vol (2004) 40:979-84. doi: 10.1016/ j.oraloncology.2004.01.012

22. Muñoz-Guerra MF, Naval-Gías L, Capote-Moreno A. Le Fort I Osteotomy, Bilateral Sinus Lift, and Inlay Bone-Grafting for Reconstruction in the Severely Atrophic Maxilla: A New Vision of the Sandwich Technique, Using Bone Scrapers and Piezosurgery. J Oral Maxillofac Surg (2009) 67:613-8. doi: 10.1016/j.joms.2008.09.004

23. Momoh AO, Yu P, Skoracki RJ, Liu S, Feng L, Hanasono MM. A Prospective Cohort Study of Fibula Free Flap Donor-Site Morbidity in 157 Consecutive Patients. Plast Reconstr Surg (2011) 128:714-20. doi: 10.1097/PRS. 0b013e318221dc2a

24. Shpitzer T, Neligan PC, Gullane PJ, Boyd BJ, Gur E, Rotstein LE, et al. The Free Iliac Crest and Fibula Flaps in Vascularized Oromandibular Reconstruction: Comparison and Long-Term Evaluation. Head Neck (1999) 21:639-47. doi: 10.1002/(SICI)1097-0347(199910)21:7<639::AIDHED8>3.0.CO;2-T

25. Valentini V, Cassoni A, Marianetti TM, Terenzi V, Fadda MT, Iannetti G. Craniomaxillofacial Fibrous Dysplasia: Conservative Treatment or Radical Surgery? A Retrospective Study on 68 Patients. Plast Reconstr Surg (2009) 123:653-60. doi: 10.1097/PRS.0b013e318196bbbe

26. Ling XF, Peng X, Samman N. Donor-Site Morbidity of Free Fibula and DCIA Flaps. J Oral Maxillofac Surg (2013) 71:1604-12. doi: 10.1016/j.joms.2013. 03.006

27. Arganbright JM, Tsue TT, Girod DA, Militsakh ON, Sykes KJ, Markey J, et al. Outcomes of the Osteocutaneous Radial Forearm Free Flap for Mandibular Reconstruction. JAMA Otolaryngol - Head Neck Surg (2013) 139:168-72. doi: 10.1001/jamaoto.2013.1615

28. Werle AH, Tsue TT, Toby EB, Girod DA. Osteocutaneous Radial Forearm Free Flap: Its Use Without Significant Donor Site Morbidity. Otolaryngol Head Neck Surg (2000) 123:711-7. doi: 10.1067/mhn.2000.110865

29. Shnayder Y, Tsue TT, Toby EB, Werle AH, Girod DA. Safe Osteocutaneous Radial Forearm Flap Harvest With Prophylactic Internal Fixation. Craniomaxillofac Trauma Reconstr (2011) 4:129-36. doi: 10.1055/s-0031-1279675

30. Zenn MR, Hidalgo DA, Cordeiro PG, Shah JP, Strong EW, Kraus DH. Current Role of the Radial Forearm Free Flap in Mandibular Reconstruction. Plast Reconstr Surg (1997) 99:1012-7. doi: 10.1097/00006534-19970400000014

31. Sinclair CF, Gleysteen JP, Zimmermann TM, Wax MK, Givi B, Schneider D. Assessment of Donor Site Morbidity for Free Radial Forearm Osteocutaneous Flaps. Microsurgery (2012) 32:255-60. doi: 10.1002/ micr. 21950

32. Martin I, Jakob M, Schaefer DJ. From Tissue Engineering to Regenerative Surgery. EBioMed Vol (2018) 28:11-2. doi: 10.1016/j.ebiom.2018.01.029

33. Hanna I, Tran H, Swope J, Peleg M. Tissue Engineering Techniques for the Reconstruction of the Atrophic and Local Maxillary Defect. In: Regenerative Strategies for Maxillary and Mandibular Reconstruction. Cham: Springer (2019). p. 87-108. doi: 10.1007/978-3-319-93668-0_8

34. Warnke P, Springer I, Wiltfang PJ, Acil PY, Eufinger PH, Wehmöller M, et al. Growth and Transplantation of a Custom Vascularised Bone Graft in a Man. Lancet (2004) 364:766-70. doi: 10.1016/S0140-6736(04)16935-3

35. Wiltfang J, Rohnen M, Egberts JH, Lützen U, Wieker H, Açil Y, et al. Man as a Living Bioreactor: Prefabrication of a Custom Vascularized Bone Graft in the Gastrocolic Omentum. Tissue Eng - Part C Methods (2016) 22:740-6. doi: 10.1089/ten.tec.2015.0501

36. DeCesare G, Deleyiannis F, Losee J. Reconstruction of Osteomyelitis Defects of the Craniofacial Skeleton. Semin Plast Surg (2009) 23:119-31. doi: 10.1055/ s-0029-1214164

37. Anderson JM, Rodriguez A, Chang DT. Foreign Body Reaction to Biomaterials. Semin Immunol Vol (2008) 20:86-100. doi: 10.1016/ j.smim.2007.11.004 
38. Ariane Christie S, Kornblith LZ, Howard BM, Conroy AS, Kunitake RC, Nelson MF, et al. Characterization of Distinct Coagulopathic Phenotypes in Injury: Pathway-Specific Drivers and Implications for Individualized Treatment. J Trauma Acute Care Surg (2017) 82:1055-62. doi: 10.1097/TA.0000000000001423

39. Yoshimura K, Suga H, Eto H. Adipose-Derived Stem/Progenitor Cells: Roles in Adipose Tissue Remodeling and Potential Use for Soft Tissue Augmentation. Regen Med (2009) 4:265-73. doi: 10.2217/17460751.4.2.265

40. Eto H, Suga H, Matsumoto D, Inoue K, Aoi N, Kato H, et al. Characterization of Structure and Cellular Components of Aspirated and Excised Adipose Tissue. Plast Reconstr Surg (2009) 124:1087-97. doi: 10.1097/PRS. 0b013e3181b5a3f1

41. Fraser JK, Wulur I, Alfonso Z, Hedrick MH. Fat Tissue: An Underappreciated Source of Stem Cells for Biotechnology. Trends Biotechnol (2006) 24:150-4. doi: 10.1016/j.tibtech.2006.01.010

42. Pradhan BB, Bae HW, Dawson EG, Patel VV, Delamarter RB. Graft Resorption With the Use of Bone Morphogenetic Protein: Lessons From Anterior Lumbar Interbody Fusion Using Femoral Ring Allografts and Recombinant Human Bone Morphogenetic Protein-2. Spine (Phila Pa 1976) (2006) 31:277-84. doi: 10.1097/01.brs.0000216442.12092.01

43. Vaidya R, Weir R, Sethi A, Meisterling S, Hakeos W, Wybo CD. Interbody Fusion With Allograft and rhBMP-2 Leads to Consistent Fusion But Early Subsidence. J Bone Jt Surg - Ser B (2007) 89:342-5. doi: 10.1302/0301-620X. 89B3.18270

44. Seeherman HJ, Berasi SP, Brown CT, Martinez RX, Sean Juo Z, Jelinsky S, et al. A BMP/activin A Chimera Is Superior to Native BMPs and Induces Bone Repair in Nonhuman Primates When Delivered in a Composite Matrix. Sci Transl Med (2019) 11. doi: 10.1126/scitranslmed.aar4953

45. Kamiya N, Ye L, Kobayashi T, Mochida Y, Yamauchi M, Kronenberg HM, et al. BMP Signaling Negatively Regulates Bone Mass Through Sclerostin by Inhibiting the Canonical Wnt Pathway. Development (2008) 135:3801-11. doi: 10.1242/dev.025825

46. Huiskes R, Rulmerman R, Van Lenthe GH, Janssen JD. Effects of Mechanical Forces on Maintenance and Adaptation of Form in Trabecular Bone. Nature (2000) 405:704-6. doi: 10.1038/35015116

47. Wang J-S, Tagil M, Isaksson H, Bostrom M, Lidgren L. Tissue Reaction and Material Biodegradation of a Calcium Sulfate/Apatite Biphasic Bone
Substitute in Rat Muscle. J Orthop Transl (2016) 6:10-7. doi: 10.1016/ j.jot.2015.11.002

48. Little DG, McDonald M, Bransford R, Godfrey CB, Amanat N. Manipulation of the Anabolic and Catabolic Responses With OP-1 and Zoledronic Acid in a Rat Critical Defect Model. J Bone Miner Res (2005) 20:2044-52. doi: 10.1359/ JBMR.050712

49. Harding AK, W-Dahl A, Geijer M, Toksvig-Larsen S, Tgil M. A Single Bisphosphonate Infusion Does Not Accelerate Fracture Healing in High Tibial Osteotomies. Acta Orthop (2011) 82:465-70. doi: 10.3109/17453674. 2011.594231

50. Chen R, Schlossman A, Breuer E, Häagele G, Tillmann C, Van Gelder JM, et al. Long-Chain Functional Bisphosphonates: Synthesis, Anticalcification, and Antiresorption Activity. Heteroat Chem (2000) 11:470-9. doi: 10.1002/ 1098-1071(2000)11:7<470::AID-HC5>3.0.CO;2-P

51. Kamiya N. The Role of BMPs in Bone Anabolism and Their Potential Targets SOST and DKK1. Curr Mol Pharmacol (2012) 5:153-63. doi: 10.2174/ 1874467211205020153

Conflict of Interest: The authors declare that the research was conducted in the absence of any commercial or financial relationships that could be construed as a potential conflict of interest.

Publisher's Note: All claims expressed in this article are solely those of the authors and do not necessarily represent those of their affiliated organizations, or those of the publisher, the editors and the reviewers. Any product that may be evaluated in this article, or claim that may be made by its manufacturer, is not guaranteed or endorsed by the publisher.

Copyright (c) 2021 Ismail, Haumer, Lunger, Osinga, Kaempfen, Saxer, Wixmerten, Miot, Thieringer, Beinemann, Kunz, Jaquiéry, Weikert, Kaul, Scherberich, Schaefer and Martin. This is an open-access article distributed under the terms of the Creative Commons Attribution License (CC BY). The use, distribution or reproduction in other forums is permitted, provided the original author(s) and the copyright owner(s) are credited and that the original publication in this journal is cited, in accordance with accepted academic practice. No use, distribution or reproduction is permitted which does not comply with these terms. 